\title{
Development of Three-Dimensional Endoscopic Ultrasound System with Optical Tracking
}

\author{
Naoshi Koizumi ${ }^{1}$, Kazuki Sumiyama ${ }^{2,3}$, Naoki Suzuki ${ }^{2}$, \\ Asaki Hattori ${ }^{2}$, Hisao Tajiri ${ }^{4}$, and Akihiko Uchiyama ${ }^{1}$ \\ ${ }^{1}$ Sch. of Sci. \& Eng., Waseda University \\ 3-4-1 Okubo Shinjuku-ku, Tokyo, Japan \\ \{koizumi,prof\}@uchiyama.comm.waseda.ac.jp \\ ${ }^{2}$ Institute for High Dimensional Medical Imaging, Jikei Univ. Sch. of Medicine \\ 4-11-1 Izumihoncho Komae-shi, Tokyo, Japan \\ \{nsuzuki, hat,kaz_sum\}@jikei.ac.jp \\ ${ }^{3}$ Dept. of Surg, Jikei Üniv. Sch. of Medicine \\ 3-25-8 Nishi-Shinbashi Minato-ku, Tokyo, Japan \\ ${ }^{4}$ Dept. of Endoscopy, Jikei Univ. Sch. of Medicine \\ 3-25-8 Nishi-Shinbashi Minato-ku, Tokyo, Japan \\ tajiri@jikei.ac.jp
}

\begin{abstract}
We have developed a new three-dimensional (3D) endoscopic ultrasound system (EUS) with convex scanning echoendoscope to diagnose and navigate by using 3D image. We use an optical tracking system which is shaped like a ribbon to detect the position of the probe and to monitor the shape of the scope inside the body. The optical tracking system can measure bends and twists at each position along with the echoendoscope. Then, the position of the tip of the echoendoscope is allotted to a $2 \mathrm{D}$ image, and the system can reconstruct and visualize a 3D image in real-time. We have reported results of our experimental studies using phantom and 3D images of vessels in animal studies.
\end{abstract}

\section{Introduction}

3D-EUS system with spiral scanning has been used as one of the diagnostic imaging modalities in Gastroenterology [1-2]. But it is difficult to use 3D images effectively in real-time and it is impossible to navigate various therapeutic and diagnostic procedures with EUS [3]. As the technological advances in EUS have expanded its applications, a new way of diagnosis using the 3D image and navigation system of endoscopic puncture would be needed. Thus, we have been developing a new 3D-EUS system with a convex scanning echoendoscope to perform various procedures with 3D image.

\section{Methods}

\subsection{Instruments}

EUS was performed with a convex scanning echoendoscope (FG32UA PENTAX) equipped with transducers ranging 5 to $7 \mathrm{MHz}$ and a display unit (EUB525 
HITACHI). Gray-scale and color Doppler video image data are fed into a personal computer (PC) (Precision 420 DELL) equipped with a video capture board (GVVCP2M/PCI IO-DATA). To detect the position of the probe inside the body, we used an optical tracking system (Shapetape Measurand) and electromagnetic tracking system (Fastrak Polhemus). Shapetape whose shape was like a ribbon was attached along with the echoendoscope form controlling head side to the tip of the echoendoscope (Figure 1). The tip of the ribbon was fixed to the tip of the echoendoscope, and the ribbon could move smoothly between echoendoscope and surrounding tube. And Fastrak was fixed to the controlling head side. 3D reconstruction and visualization are performed with the above-mentioned PC.

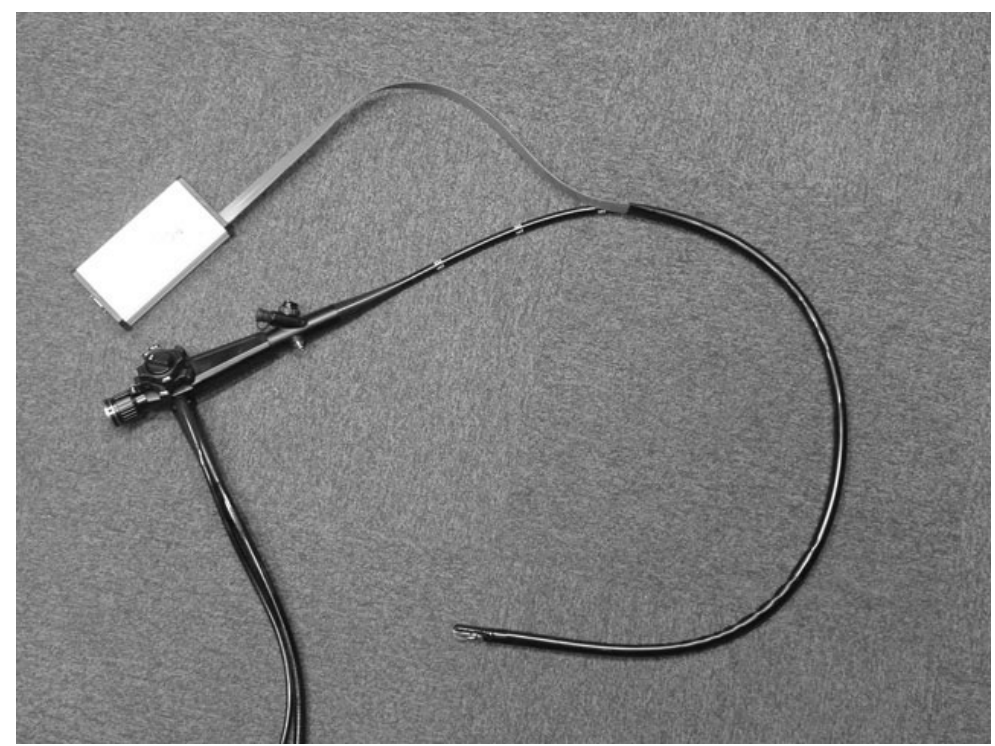

Fig. 1. Convex scanning echoendoscope with optical tracking system

\subsection{D Reconstruction and Visualization in Real-Time}

In this system, the whole shape and relative position from the controlling head side to the tip of the echoendoscope are acquired by optical tracking system. The optical tracking system is composed of an array of fiber optic sensors (Figure 2), and can measure bends and twists at each position of the ribbon (Figure 3).

To calculate the twist and bend of each position from controlling head side to the tip, the whole shape of the ribbon is detected. In each position of the optical tracking system, there are three situations of coordinate systems. One is the original coordinate system before twist and bend $\left(\Sigma_{\mathrm{o}}\right)$. Next is the coordinate system after rotating twist $(\mathrm{t}),\left(\Sigma_{\mathrm{t}}\right)$. Finally there is the coordinate system after rotating twist and bend $(\mathrm{b}),\left(\Sigma_{\mathrm{b}}\right)$. Then the rotation matrix from $\Sigma_{\mathrm{o}}$ to $\Sigma_{\mathrm{t}},\left({ }^{\mathrm{o}} \mathrm{R}_{\mathrm{t}}\right)$, is defined as follows: 


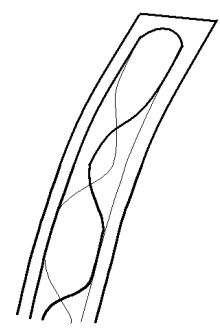

Fig. 2. Inner structure of the ribbon
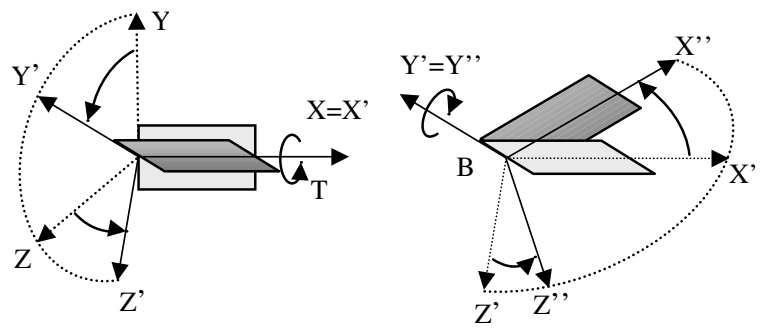

Fig. 3. The definition of the twist and bend

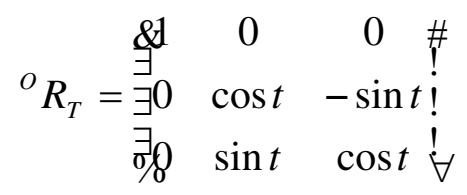

In the same way, the rotation matrix from $\Sigma_{\mathrm{t}}$ to $\Sigma_{\mathrm{b}},\left(\mathrm{R}_{\mathrm{b}}\right)$, is defined as follows:

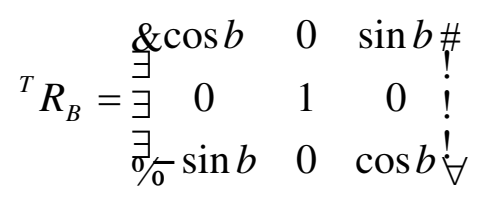

Then the rotation matrix from $\Sigma_{\mathrm{o}}$ to $\Sigma_{\mathrm{b}},\left({ }^{\circ} \mathrm{R}_{\mathrm{b}}\right)$, is defined as follows:

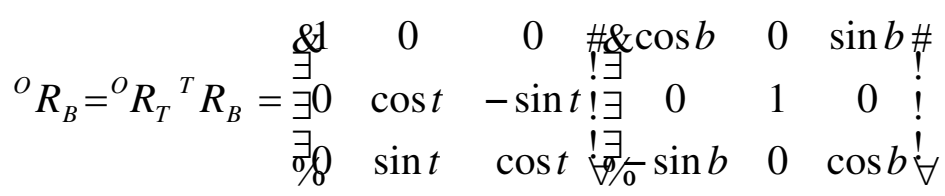

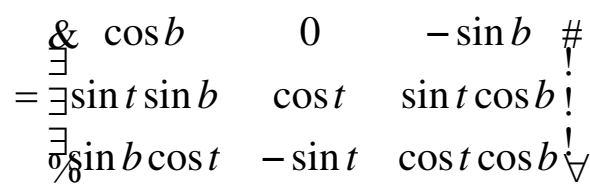

To use this rotation matrix, positions of each position of the ribbon are calculated. This rotation matrix calculates the number $(n, 1 \sim N)$ of twists and bends $\left({ }^{\circ} R_{b}[n]\right)$. The position of the number $\mathrm{n}$ from origin of the ribbon (X[n], Y[n], Z[n]) is calculated as follows:

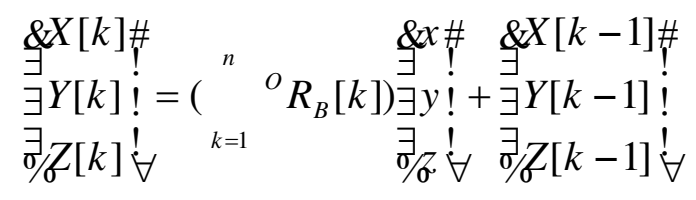


In above-mentioned equation, $(\mathrm{x}, \mathrm{y}, \mathrm{z})$ represented the position of the ribbon in $\Sigma_{\mathrm{o}}$ of number $n=1$. In addition to this relative position acquired by the optical tracking system, in measuring the original position of the ribbon with the electromagnetic tracking system can detect the position of the probe in world space coordinate system. These positions which are calculated are allotted to 2D image, and 3D image is reconstructed and visualized in real-time. Also, the display of our system can show the 2D image which is being scanned and the shape of the echoendoscope which is being monitored by optical tracking system and 3D image. A block diagram of the key components is shown in Figure 4.

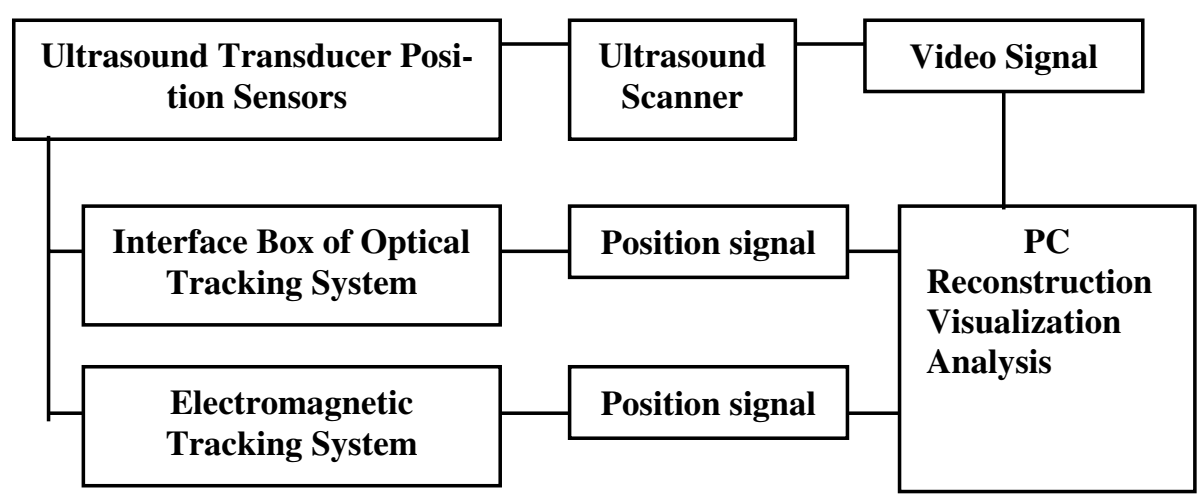

Fig. 4. A block diagram of the key components of the system

\section{Results}

Our system applied to experimental and animal studies. In experimental studies using a phantom of spherical shape, our system could reconstruct and visualize it in realtime and to observe the reconstructed 3D image, the shape of it could be confirmed as a sphere. (Figure 5). And to display the position of the 2D image that is scanning inside 3D image, the user could observe the 2D image with its surrounding information. Since our system can confirmed the updated 3D image as scanning 2D image, in the case of fail in scanning, the user could scan again speedy. In experimental studies, the characteristic of the optical tracking system and procedures in scanning were examined and confirmed. In animal studies, our system could reconstruct and visualize the portal vein of the hepatic hilum of the pig in real-time (Figure 6). In Figure.6, the upper left sub window shows $2 \mathrm{D}$ images which is scanning, and the frame that is composed of green and red lines show the region of interest (ROI). In Figure.6, the right window shows 3D image reconstructed by using ROI in $2 \mathrm{D}$ image. In Figure.6, lower left window shows the shape of the echoendoscope constructed by the data from optical tracking system, and to monitor and display the shape of echoendoscope 
inside body of animal, the operator could recognize the areas which were scanned intuitively.

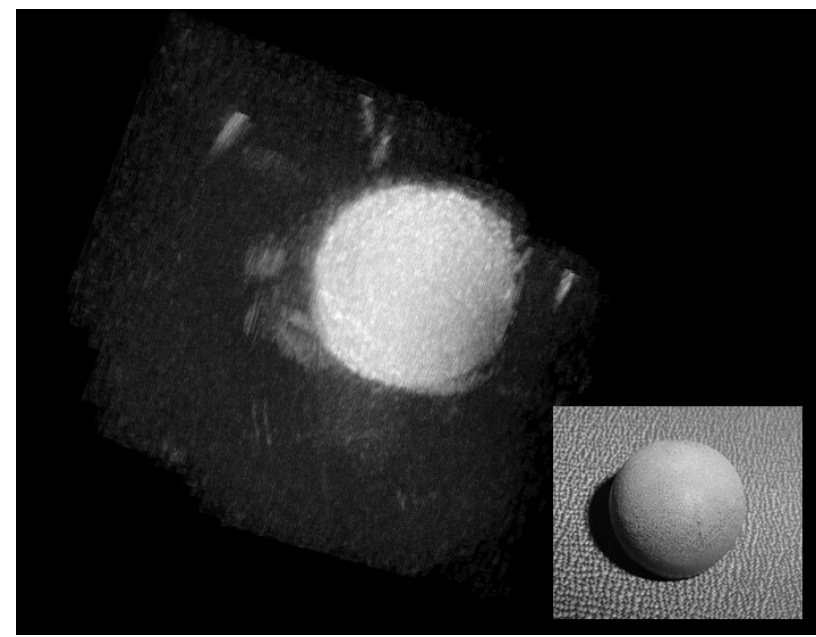

Fig. 5. Phantom and reconstructed 3D image in experimental studies

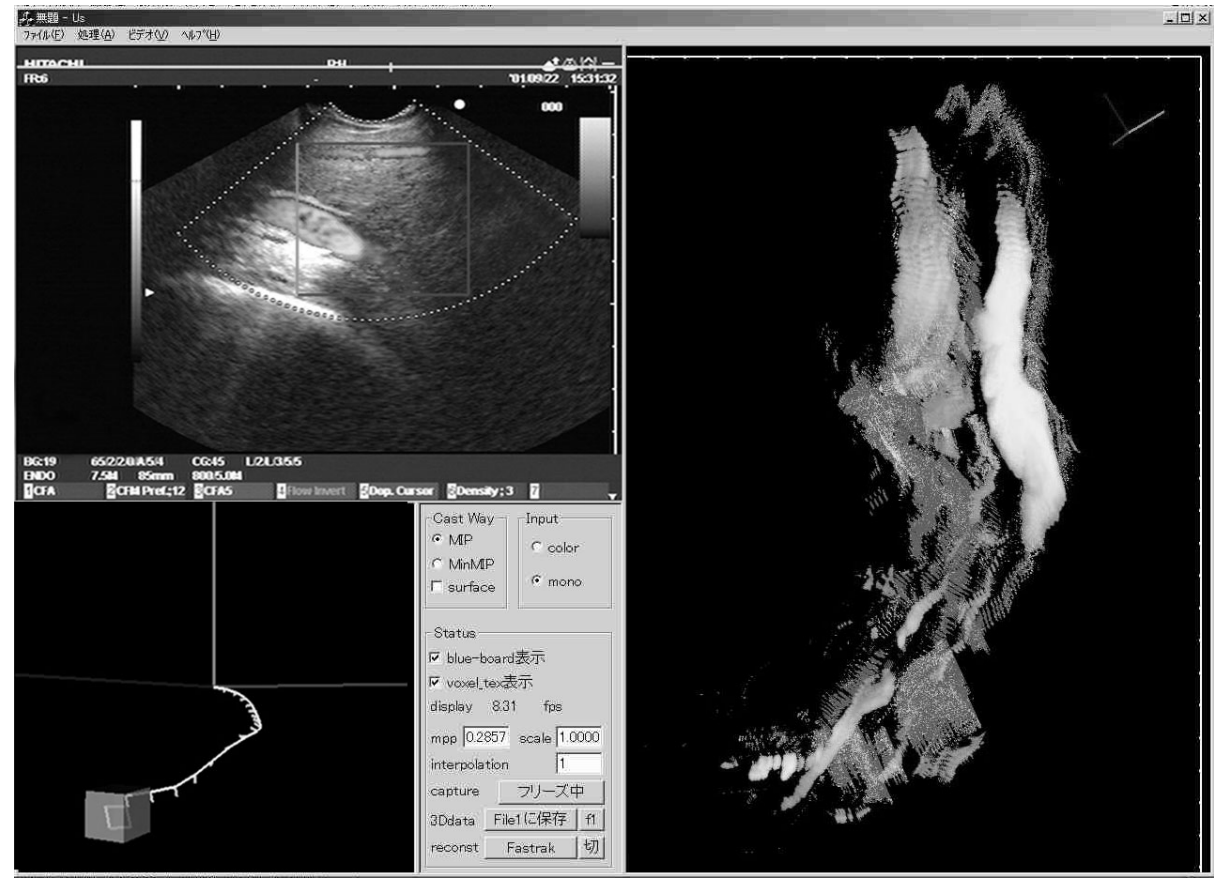

Fig. 6. Display of the system in animal studies. Upper left sub window shows Power Doppler sonography, and lower left shows the shape of echoendoscope inside body. The right window shows a real-time $3 \mathrm{D}$ image that was reconstructed 


\section{Discussion}

According to the improvement of various medical imaging modalities, 3D images have been used in diagnosis and navigation field. In ultrasound, 3D images have been used in the obstetrics and gynecology field and the navigation system in open surgery of the liver and so on [4-6]. However in EUS, because of the difficulty in detecting the position of the probe inside the body, it is difficult to develop a system which makes good use of 3D images. In this study, our system established a foundation of $3 \mathrm{D}$ reconstruction and visualization in real-time using a convex scanning echoendoscope, and suggested the possibility of a navigation system of endoscopic punctures. Now, the problems of our system are ones of accuracy and ways of attaching to the optical tracking system. These kind of problems will be solved by combining it with our system and burying the optical fiber structure into the echoendoscope.

In summary, we developed new 3D-EUS system using a convex scanning echoendoscope with an optical tracking system. This system can reconstruct and visualize 3D image in real-time and monitor the shape of the echoendoscope inside the body. Experimental and animal studies demonstrated the technical feasibility of this system. We believe that further improvement of this system will be able to expand applications of 3D-EUS.

\section{Reference}

1. Yoshino J, Nakazawa S, Inui K, Katoh Y, Wakabayshi T, Okushima T, Kobayashi T and Watanabe S: Surface-rendering imaging of gastrointestinal lesions by three-dimensional endoscopic ultrasonography. Endoscopy 1999 Sep;31(7):541-5

2. Kida M, Watanabe M, Sugeno S, et al: 3-Dimensional Endoscopic Ultrasonography for Upper Gastrointestinal Diseases. Endoscopy, 28(suppl): S38, 1996.

3. Kazuya Y, Shunichi N, Shunichiro I, Tadayoshi K, Yoshihiro S: Imaging of the colorectal cancer using 3D-EUS system. Early Colorectal Cancer vol.3, number 3, 1999

4. Baba K: Development of three-dimensional ultrasound in obsterics and gynecology: Technical aspects and possibilities. In 3-D Ultrasound in Obstetrics and Gynecology. Merz E ed., Lippincott Williams \& Wilkins, Philadelphia, USA, 3-8, 1998.

5. Nelson TR, Pretorius DH, Hagan-Ansert S: Fetal heart assessment using threedimensional ultrasound. J Ultrasound Med, 14(suppl): S30 1995

6. Steven c. Rose, Dolores H. Pretorius, Thomas B. Kinney, Thomas R. Nelson, Karim Valji, Horacio R. D'Agostino, Nannette M. Forsythe, Anne C. Roberts, Michael L. MancoJohnson: Three-dimensional Sonographic Guidance for Transvenous Intrahepatic Invesive Procedures. Feasibility of a New Technique. JVIR 1999; 10:189-198 